\title{
DRUG RESISTANCE MECHANISMS ON COLORECTAL CANCER
}

\author{
Hasan Kurter ${ }^{1}$, Janberk Yeşil ${ }^{2,3}$, Ezgi Daskin ${ }^{1}$, Gizem Calibasi-Kocal', \\ Hulya Ellidokuz ${ }^{5}$, Yasemin Basbinar, ${ }^{4}$
}

\author{
${ }^{1}$ Dokuz Eylul University, Institute of Health Sciences, Department of Translational Oncology, Izmir, Turkey. \\ ${ }^{2}$ Eyuboglu College, İstanbul, Turkey. \\ ${ }^{3}$ Dokuz Eylul University, Institute of Oncology, Translational Oncology Pre-graduate Research Group, Izmir, Turkey. \\ ${ }^{4}$ Dokuz Eylul University, Institute of Oncology, Department of Translational Oncology, Izmir, Turkey. \\ ${ }^{5}$ Dokuz Eylul University, Institute of Oncology, Department of Preventive Oncology, Izmir, Turkey.
}

Address for Correspondence: Yasemin Basbinar E-mail: yasemin.baskin@deu.edu.tr Received: 07.01.2021; Accepted: 31.01.2021; Available Online Date: 04.03.2021

(C) Copyright 2021 by Dokuz Eylül University, Institute of Health Sciences - Available online at https://dergipark.org.tr/en/pub/jbachs

Cite this article as: Kurter $\mathrm{H}$, Yeşil J, Daskin E, Calibasi-Kocal G, Ellidokuz H, Basbinar Y. Drug Resistance Mechanisms on Colorectal Cancer. J Basic Clin Health Sci 2021; 1: 88-93

\begin{abstract}
Standard treatment for colorectal cancer includes surgery, radiotherapy, and chemotherapy. Conventional chemotherapeutic agents used in colorectal cancer such as 5-fluorouracil, capecitabine which is oral form of 5-fluorouracil, irinotecan, and oxaliplatin. One of the major challenges in chemotherapy considered as drug resistance. Drug resistance occurs in many different mechanisms such as alteration in tumor microenvironment, growth kinetics, genetic variations, and tumor heterogeneity. These cytotoxic agents are used in combination form to overcome drug resistance on colorectal cancer. They can also be used in combination with cytotoxic agents as bevacizumab and cetuximab. Combination therapy depends on tumor burden, type, stage, and molecular characteristics. It is crucial to choose the combination therapy to be applied by taking these factors into consideration. In this review, current drug resistance mechanisms were discussed and then mechanisms of conventional chemotherapy related resistance were explained.
\end{abstract}

Keywords: Drug resistance, colorectal cancer, chemotherapy, drug combinations.

\section{INTRODUCTION}

The basic treatments of colorectal cancer (CRC) include surgery, radiotherapy, and pharmacotherapy. Pharmacotherapy is divided into two classes as biologic agents and cytotoxic agents. The combination of biologic agents, such as bevacizumab (VEGF inhibitor), and cetuximab (EGFR inhibitor) have been some benefits on metastatic CRC. On the other hand, 5-fluorouracil (5-FU), irinotecan, oxaliplatin, and capecitabine are used as cytotoxic agents in CRC treatment (1). They work in conjunction with one another as combination therapy. For instance, FOLFIRI (5-FU/leucovorin/irinotecan), FOLFOXIRI (5-FU/leucovorin/oxaliplatin/irinotecan) FOLFOX (5-FU/leucovorin/oxaliplatin), and CAPOX (capecitabine/oxaliplatin) are FDA approved combination drugs and these agents can use with biological agents. Unfortunately, drug resistance can develop during to drug applications of CRC or other cancers, and this limits the effectiveness of drugs (2). 
Drug resistance is the main handicap in the treatment of cancer or other diseases. There are many factors that trigger drug resistance. These factors affect drug pharmacokinetics such as drug inactivation, alteration of drug targets, escape from apoptosis, increasing DNA-damage repair mechanism etc. This resistance can classify into two main categories as intrinsic resistance and acquired drug resistance (3). Intrinsic resistance refers to inherent resistance that exists before drug treatment. Inherent genetic mutations, tumor heterogeneity, and some signal pathways that caused the inactivation of cancer drugs can be the main reason for intrinsic resistance (4). Acquired drug resistance can gradually develop during drug treatment, thus effectiveness of the drug reduces. The formation of new proto-oncogenes, mutations on the genes of drug targets and changes in tumor microenvironment can cause acquired drug resistance. In this review, we will explain of drug resistance mechanisms and mechanisms of using cytotoxic drugs in CRC treatment (4).

\section{DRUG RESISTANCE MECHANISMS}

\section{Tumor Size and Growth Kinetics}

Nearly all solid cancer types have an inverse relationship between tumor size and curability. Enhancement of tumor size causes increase on metastatic character and drug resistance (5). GoldieColdman hypothesis declares the relationship of curability and tumor size. According to this hypothesis, larger tumors are more capable to contain drug-resistant clones (6). Although, this hypothesis has not been confirmed on clinical approaches in uniform manner and further complications such as drug toxicity that is necessary to be considered. The relationship between tumor size and drug resistance is highly related to cell growth kinetics. One of the most important matter on tumor size and regression after treatment is the Norton-Simon hypothesis. The hypothesis suggests that solid cancer cells proliferate in a sigmoidal curve until they reach a certain size then proliferation rate reduces while tumor burden increases (7). Chemotherapeutic agents reduce tumor size and affect growth kinetics. Tumor size is reduced after the first dose of chemotherapy and also, the change of growth kinetics causes exponential growth in manner of time. From this point of view, effectiveness of chemotherapeutics is augmented by shortening the time between simultaneous doses.

\section{Tumor Heterogeneity}

Cancer cells keep evolving and changing even after malignant transformation. This molecular evolution causes to obtain heterogeneity and drug resistance of cancer cells via undergoing changes at various molecular levels. Tumor heterogeneity process involves several differentiations in terms of epigenetics and genetics these are cell morphology, metabolism, proliferation, motility (8). Tumor heterogeneity is subdivided into two classes called intratumoral and intertumoral heterogeneity. Intertumoral heterogeneity refers to genetic variations of a tumor tissue which has same histological feature on different patients (9). On the other hand, intratumoral heterogeneity involves a combination of cancer cells which evolved from primer cancer cell via genetic variations to gain metastatic character and develop drug resistance (10). Gausachs et. al shows that mutational heterogeneity in KRAS and APC could cause polyclonality in early colorectal carcinogenesis (11). Thus, polyclonality induces the possibility of drug-resistance clones in tumor tissue.

\section{Tumor Microenvironment}

Tumor microenvironment (TME) contains important components that provide nutrition and development of tumor tissue. These components include extracellular factors such as blood vessels, endothelial, myeloid, lymphoid, and growth factors, soluble factors, extracellular matrix, $\mathrm{pH}$, oxygenation, and stromal cells. The effect of tumor microenvironment on drug resistance is highly related to the cancer cells and TME components responses to drugs (12). Thus, tumor-cancer cells, tumorstromal cells and tumor-ECM communication have an important effect on the development of drug resistance. Although, growth factors such as platelet - derived growth factors (PDGFs), fibroblast growth factors (FGFs), vascular endothelial growth factors (VEGFs), insulin-like growth factors (IGFs) play enormous role on drug resistance via providing essential signals for proliferation, hence play important role on tumor burden augmentation. Angiogenic factors also have a great influence on drug resistance. Pro-angiogenic factors such as 
FGFs and PDGFs, create resistance to anti-VEGF / VEGFR drugs which are commonly used in renal carcinoma treatment. Hypoxic environment and inflammatory factors also have an important effect on tumor heterogeneity and cause drug resistance. Increased cancer associated fibroblasts (CAFs) cause drug resistance on cancer therapy via triggering accumulation of extracellular matrix, interleukin, and cytokines secretion. PI3/Akt, JAK/STAT and Ras-MAPK pathways are responsible on inhibition of apoptosis and increased interleukin 6 secretion activates those pathways and causes drug resistance. Furthermore, non-cellular components of extracellular matrix such as low $\mathrm{pH}$, hypoxia, stiffness of extracellular matrix and hypoxia play important role on drug resistance (13).

\section{DRUG RESISTANCE IN COLORECTAL CANCER}

5-fluorouracil, an effective antimetabolite and cell cycle targeting drug, is used in the treatment of many lots of cancer such as CRC, breast cancer, and pancreatic cancer (14). 5-FU inhibits the thymidylate synthase (TS) expression and arrests DNA replication via binding its metabolites to DNA [15]. Thus, it demonstrates the toxic effect on cancer cells. The resistance of 5-FU is closely connected to drug metabolism, drug absorption, and changed in TS expression (16).

Overexpression of TS is one of the major 5-FU resistance reasons for in vitro and in vivo studies, as well as in clinic While the presence of pre-treatment high TS level causes internal resistance, the increase in gene amplification of TS and mutations in its structure during the treatment cause acquired resistance. After the 5-FU treatment, the overall survival rate of patients with an elevated level of TS is shorter than in a patient with a low level of TS (17). The other enzymes such as uridine kinase, orotate phosphoribosyltransferase (responsible for thymidine biosynthesis), and thymidine kinase are closely related to reverse the $5-\mathrm{FU}$ resistance. In other words, the expression levels of these enzymes are lower in 5-FU resistance cells (18). Besides, decrease of monophosphate kinase expression (UMPK), which is involved in the activation of 5fluorouridine triphosphate (5-FUTP) and binds to RNA, was also observed in resistant cells (19).

Thymidine phosphorylase (TP) has two important roles in metabolism. Primarily, TP converts thymidine to thymine and 2-deoxyribose-1-phosphate. Also, TP induces angiogenesis. Therefore, TP overexpression is related to malignancy, tumor aggressiveness, and poor prognosis (20). In this context, TP is regularly overexpressed in tumor tissue and some prodrugs such as capecitabine were developed for bioactivation of TP. Capecitabin is the first oral drug form for using CRC treatment. It is metabolized and transformed to 5'-deoxy-5- fluorouridine (5'-DFUR) and 5'-deoxy-5-fluorocytidine (5'-OFCR). Then, 5'DFUR is hydrolyzed to 5-FU via TP and performs cytotoxic effect on cancer cells. Drug resistance mechanism of 5-FU is similar to capecitabine (21). Notably, TP is an important enzyme for transformation of the capecitabine to 5-FU and it plays a key role on the drug resistance. The elevated expression of TP is related to better response to capecitabine while low expression of TP refers to drug resistance (22).

Irinotecan (CPT-11) is a semisynthetic analog of camptothecin that is isolated from the Camptotheca acuminate tree. CPT-11 is used in treatment of many various cancer such as lung cancer, pancreatic cancer, and CRC (23). CPT-11 is not only used as a monotherapy in cancer treatment but also it is used with other cytotoxic agents like 5-FU and oxaliplatin. Therewithal, it also can be used with monoclonal antibodies such as bevacizumab and cetuximab (24). CPT-11 is a cytotoxic agent that inhibits topoisomerase I, selectively. CPT-11 is hydrolyzed to 7-ethyl-10-hydroxycamptothecin (SN-38), which is the active form of CPT-11, via carboxylesterase. This biotransformation involves the elimination of the $\mathrm{C} 10$ group from CPT-11 and then become SN-38 (21). SN-38 demonstrates a stronger cytotoxic activity than CPT-11 (25). CPT-11 or its active form SN-38 creates the TOPO I-irinotecan-DNA complex for breaking stranded DNA (26).

Irinotecan has some drug resistance mechanism. These resistance mechanisms are changes in the expression levels of enzymes responsible for the transformation of irinotecan to its active form SN-38, increasing expression of drug efflux proteins, decreasing expression of topoisomerase 1, and occurring mutations in the structure of topoisomerase 1 (27).

Formation of the TOPO1-irinotecan-DNA complex causes DNA breaks. However, prevention of this 
complex develops drug resistance. The mutations in the TOPOI, decreasing expression ofTOPO1, and interaction of TOPO1 with some proteins are the reasons for the resistance (28). CPT-11 is taken into the body passively and it is hydrolyzed to active metabolite form SN-38 by carboxylesterases 1 and 2 (CES1-2) in the liver. CPT-11 can be converted into its inactive metabolites NPC (7-ethyl-10-(4-amino-1piperidino) carbonyloxycamptothecin) and APC (7ethyl-10-[4-N-(5-aminopentanoic acid)-1-piperidino] carbonyloxycamptothecin) by cytochrome P450 isoform enzymes (CYP3A4, CYP3A5). Decrease of CES1-2 and increase of CYP3A4/ 5 are related to resistance development to irinotecan. Increased CES1-2 enzyme activity increases SN-38 amount. However, increased CYP3A4/ 5 enzyme activity degrades irinotecan, resulting in formation of increased inactive form (23). At the same time, ATPcassette protein, $A B C B 1$, P-glycoprotein, can pump the irinotecan and $\mathrm{SN}-38$ outside of the cell which causes reduction of the drug accumulation in cancer cell (29). SN-38 is converted via uridine diphosphate glucuronosyltransferase 1As (UGT1As) to the inactive form SN-38-glucuronide (SN-38G). Then, it can be converted back into the active form by $\beta$ glucosidase, a bacterial enzyme which is found in the gut (30).

Oxaliplatin that is a third-generation platinum drug has 1,2-diaminocyclohexane (DACH) ligand and a separated oxalate group. Biotransformation of oxaliplatin occurs spontaneously and non-enzymatic reaction in the biological fluid. Firstly, the oxalate group replaces with $\mathrm{H} 2 \mathrm{O}, \mathrm{Cl}$, and $\mathrm{HCO} 3-$ ions, and forming dichloro-, monochloro-, and diaquo-DACH platin active groups bind to DNA afterward. These active platin groups bind to guanine-guanine or adenine-guanine intrastrand adducts (31).

The resistance mechanism of oxaliplatin is related to nucleotide excision repair (NER), drug efflux protein, glutathione metabolism, and copper transporter proteins. NER is the main mechanism in oxaliplatin resistance. While xeroderma pigmentosum group $A$ $(X P A)$ and excision repair cross-complementing group 1 (ERCC1) gene expression in the NER mechanism increases, the cytotoxic effect of oxaliplatin decreases (32).

As the drug accumulation within the cell decreases, the sensitivity of the cells to the drug decreases and resistance develops. ABC-transporter proteins are mostly responsible for this resistance mechanism. When oxaliplatin conjugates with glutathione, the level of MRP2 expression increases. Therefore, MRP2 has an important role in drug resistance (33). Solid carrier membrane proteins are expressed to maintain metabolism balance of the cell by mediating the transport of exogenous and endogenous substances. Decreased expression levels of, hMATE1 (SLC47A1), hOCT3 (SLC22A3), and hMATE2-K (SLC47A2) are responsible from oxaliplatin transport, resulting in oxaliplatin resistance (34). Apart from the above, transforming growth factor $\beta 1$ (TGF- $\beta 1$ ) is secreted by cells within in tumor microenvironment. Excreted TGF- $\beta 1$ induces drug resistance by helping epithelial to mesenchymal transition (35).

\section{CONCLUSION}

CRC cases are constantly increasing as much as other cancer types. In addition to surgery and radiotherapy, chemotherapeutics is also used for CRC treatment. However, intrinsic or acquired drug resistance are likely the most challenging factor in the chemotherapy process. There are lots of aspects included in drug resistance development. Drug inactivation, drug efflux proteins, drug target alteration, increase in DNA damage repair mechanisms, tumor microenvironment and tumor heterogeneity and growth kinetics of cancer cells are the main causes of drug resistance. Combination therapy is an effective method to overcome drug resistance. However, using drug combinations on cancer treatment involves handicaps such as genetic variations and tumor specificity for each individual patient. Combination therapy must occur minimum toxicity on healthy tissue while performing cytotoxic effect on tumor tissue. From this point of view, the mechanisms of candidate drugs and genetic variation, tumor microenvironment components, tumor burden, and growth kinetics of the tumor must be well-known.

Acknowledgements: This work was supported by Dokuz Eylul University, Scientific Research Projects (Project Number: 2020.KB.SAG.039).

Conflict of Interest: No conflict of interest was declared by the authors.

Peer-review: Externally peer-reviewed. 


\section{REFERENCES}

1. Longley DB, Allen WL, Johnston PG. Drug resistance, predictive markers and pharmacogenomics in colorectal cancer. Biochim Biophys Acta. 2006; 1766(2):184-196.

2. Dekker E, Tanis PJ, Vleugels JLA, Kasi PM, Wallace MB. Colorectal cancer. Lancet. 2019; 394(10207):1467-1480.

3. Vasan N, Baselga J, Hyman DM. A view on drug resistance in cancer. Nature. 2019; 575(7782):299-309.

4. Wang $X$, Zhang $H$, Chen $X$. Drug resistance and combating drug resistance in cancer. Cancer Drug Resist 2019; 2:141-160.

5. Goldie, J. H. \& Coldman, A. J. The genetic origin of drug resistance in neoplasms: implications for systemic therapy. Cancer Res 1984; 44:3643-3653.

6. Goldie JH, Coldman AJ. A mathematic model for relating the drug sensitivity of tumors to their spontaneous mutation rate. Cancer Treat Rep. 1979 ;63(11-12):1727-1733.

7. Norton, L., Simon, R., Brereton, H. D. \& Bogden, A. E. Predicting the course of Gompertzian growth. Nature 1976; 264:542545.

8. Dagogo-Jack I, Shaw AT. Tumour heterogeneity and resistance to cancer therapies. Nat Rev Clin Oncol. 2018; 15(2):8194.

9. Sutherland KD, Visvader JE. Cellular Mechanisms Underlying Intertumoral Heterogeneity. Trends Cancer. 2015; 1(1):1523.

10. Hinohara K, Polyak K. Intratumoral Heterogeneity: More Than Just Mutations. Trends Cell Biol. 2019; 29(7):569-579.

11. Gausachs M, Borras E, Chang K, et al. Mutational Heterogeneity in APC and KRAS Arises at the Crypt Level and Leads to Polyclonality in Early Colorectal Tumorigenesis. Clin Cancer Res. 2017; 23(19):5936-5947.

12. Sun Y. Tumor microenvironment and cancer therapy resistance. Cancer Lett. 2016; 380(1):205-215.

13. Haider T, Pandey V, Banjare N, Gupta PN, Soni $V$. Drug resistance in cancer: mechanisms and tackling strategies. Pharmacol Rep. 2020; 72(5):1125-1151.
14. Deng J, Wang Y, Lei J, Lei W, Xiong JP. Insights into the involvement of noncoding RNAs in 5-fluorouracil drug resistance. Tumour Biol. 2017; 39(4):1010428317697553.

15. Longley DB, Harkin DP, Johnston PG. 5fluorouracil: mechanisms of action and clinical strategies. Nat Rev Cancer. 2003; 3(5):330338.

16. Cao X, Hou J, An Q, Assaraf YG, Wang X. Towards the overcoming of anticancer drug resistance mediated by p53 mutations. Drug Resist Updat. 2020; 49:100671.

17. Blondy S, David V, Verdier M, Mathonnet M, Perraud A, Christou N. 5-Fluorouracil resistance mechanisms in colorectal cancer: From classical pathways to promising processes. Cancer Sci. 2020; 111(9):31423154.

18. Fujii R, Seshimo A, Kameoka S. Relationships between the expression of thymidylate synthase, dihydropyrimidine dehydrogenase, and orotate phosphoribosyltransferase and cell proliferative activity and 5-fluorouracil sensitivity in colorectal carcinoma. Int J Clin Oncol. 2003; 8(2):72-78.

19. Humeniuk R, Menon LG, Mishra PJ, et al. Decreased levels of UMP kinase as a mechanism of fluoropyrimidine resistance. Mol Cancer Ther. 2009; 8(5):1037-1044.

20. Furukawa $T$, Tabata $S$, Yamamoto $M$, et al. Thymidine phosphorylase in cancer aggressiveness and chemoresistance. Pharmacol Res. 2018; 132:15-20.

21. Van der Jeught $K, X u$ HC, Li YJ, Lu XB, Ji G. Drug resistance and new therapies in colorectal cancer. World J Gastroenterol. 2018; 24(34):3834-3848.

22. Stark M, Bram EE, Akerman M, MandelGutfreund Y, Assaraf YG. Heterogeneous nuclear ribonucleoprotein $\mathrm{H} 1 / \mathrm{H} 2$-dependent unsplicing of thymidine phosphorylase results in anticancer drug resistance. J Biol Chem. 2011; 286(5):3741-3754.

23. $\mathrm{Xu} \mathrm{Y,} \mathrm{Villalona-Calero} \mathrm{MA.} \mathrm{Irinotecan:}$ mechanisms of tumor resistance and novel strategies for modulating its activity. Ann Oncol. 2002; 13(12):1841-1851.

24. Bailly C. Irinotecan: 25 years of cancer treatment. Pharmacol Res. 2019; 148:104398. 
25. Hicks LD, Hyatt JL, Stoddard S, et al. Improved, selective, human intestinal carboxylesterase inhibitors designed to modulate 7-ethyl-10-[4(1-piperidino)-1-piperidino]carbonyloxy camptothecin (Irinotecan; CPT-11) toxicity. J Med Chem. 2009; 52(12):3742-3752.

26. Liu LF, Duann P, Lin CT, D'Arpa P, Wu J. Mechanism of action of camptothecin. Ann N Y Acad Sci. 1996; 803:44-49.

27. de Man FM, Goey AKL, van Schaik RHN, Mathijssen RHJ, Bins S. Individualization of Irinotecan Treatment: A Review of Pharmacokinetics, Pharmacodynamics, and Pharmacogenetics. Clin Pharmacokinet. 2018; 57(10):1229-1254.

28. Gmeiner WH. Entrapment of DNA topoisomerase-DNA complexes by nucleotide/nucleoside analogs. Cancer Drug Resist. 2019; 2:994-1001.

29. Trumpi K, Emmink BL, Prins AM, et al. ABCTransporter Expression Does Not Correlate with Response to Irinotecan in Patients with Metastatic Colorectal Cancer. J Cancer. 2015; 6(11):1079-1086.

30. Takano M, Sugiyama T. UGT1A1 polymorphisms in cancer: impact on irinotecan treatment. Pharmgenomics Pers Med. 2017; 10:61-68.

31. Graham MA, Lockwood GF, Greenslade D, Brienza S, Bayssas M, Gamelin E. Clinical pharmacokinetics of oxaliplatin: a critical review. Clin Cancer Res. 2000; 6(4):1205-1218.

32. Martinez-Balibrea E, Martínez-Cardús A, Ginés $A$, et al. Tumor-Related Molecular Mechanisms of Oxaliplatin Resistance. Mol Cancer Ther. 2015; 14(8):1767-1776.

33. Burger $\mathrm{H}$, Loos WJ, Eechoute $\mathrm{K}$, Verweij J, Mathijssen RH, Wiemer EA. Drug transporters of platinum-based anticancer agents and their clinical significance. Drug Resist Updat. 2011; 14(1):22-34.

34. Gao H, Zhang S, Hu T, et al. Omeprazole protects against cisplatin-induced nephrotoxicity by alleviating oxidative stress, inflammation, and transporter-mediated cisplatin accumulation in rats and HK-2 cells. Chem Biol Interact. 2019; 297:130-140.

35. Mao L, Li Y, Zhao J, et al. Transforming growth factor- $\beta 1$ contributes to oxaliplatin resistance in colorectal cancer via epithelial to mesenchymal transition. Oncol Lett. 2017; 14(1):647-654. 